\title{
Humoral Reactivity of Renal Transplant-Waitlisted Patients to Cells From GGTA1/CMAH/B4GalNT2, and SLA Class I Knockout Pigs
}

Gregory R. Martens, MD, ${ }^{1}$ Luz M. Reyes, PhD, ${ }^{1}$ James R. Butler, MD, ${ }^{2}$ Joseph M. Ladowski, MS, ${ }^{1}$ Jose L. Estrada, DVM, PhD, ${ }^{1}$ Richard A. Sidner, PhD, ${ }^{2}$ Devin E. Eckhoff, MD, ${ }^{1,3}$ Matt Tector, PhD, ${ }^{1,3}$ and $\mathrm{A}$. Joseph Tector, MD, PhD ${ }^{1,3}$

Background. Antipig antibodies are a barrier to clinical xenotransplantation. We evaluated antibody binding of waitlisted renal transplant patients to 3 glycan knockout (KO) pig cells and class I swine leukocyte antigens (SLA). Methods. Peripheral blood mononuclear cells from SLA identical wild type (WT), a1, 3-galactosyltransferase (GGTA1) KO, GGTA1/ cytidine monophosphate-N-acetylneuraminic acid hydroxylase (CMAH) KO, and GGTA1/ CMAH /b1,4 N-acetylgalactosaminyl transferase (B4GalNT2) KO pigs were screened for human antibody binding using flow cytometric crossmatch (FCXM). Sera from 820 patients were screened on GGTA1/CMAH/B4GalNT2 KO cells and a subset with elevated binding was evaluated further. FCXM was performed on SLA intact cells and GGTA1/SLA class I KO cells after depletion with WT pig RBCs to remove cell surface reactive antibodies, but leave SLA antibodies. Lastly, human and pig reactive antibodies were eluted and tested for cross-species binding and reactivity to single-antigen HLA beads. Results. Sequential glycan $\mathrm{KO}$ modifications significantly reduce antibody binding of waitlisted patients. Sera exhibiting elevated binding without reduction after depletion with WT RBCs demonstrate reduced binding to SLA class I KO cells. Human IgG, eluted from human and pig peripheral blood mononuclear cells, interacted across species and bound single-antigen HLA beads in common epitope-restricted patterns. Conclusions. Many waitlisted patients have minimal xenoreactive antibody binding to the triple $\mathrm{KO}$ pig, but some HLA antibodies in sensitized patients cross-react with class I SLA. SLA class I is a target for genome editing in xenotransplantation.

(Transplantation 2017;101: e86-e92)

idney transplantation is limited by the shortage of suitable donors, but using pig kidneys has the potential to eliminate this problem. Unfortunately, humans have

Received 9 July 2016. Revision received 15 December 2016

Accepted 22 December 2016.

1 Department of Surgery, University of Alabama at Birmingham, Birmingham, AL.

2 Department of Surgery, Indiana University, School of Medicine, Indianapolis, IN.

${ }^{3}$ Transplant Surgery, University of Alabama at Birmingham, Birmingham, AL.

G.R.M. is supported by a research scholarship from the American College of Surgeons. A.J.T. is the founder of Xenobridge LLC, has United Therapeutics funding and applied for patents. R.A.S. receives consulting fees related to xenotransplantation.

This study was funded by Indiana University. Indiana University Transplant Institute, and United Therapeutics.

This article has been revised approved by all authors. G.R.M., R.A.S., D.E.E., M.T., A.J.T. drafted this article developed refined study concepts. L.M.R., J.L.E. performed SLA sequencing, gene manipulations, pig cloning genotyping. G.R.M., R.A.S., J.R.B., J.M.L. performed data collection data analysis.

Correspondence: A. Joseph Tector, ZRB 7011720 2nd Ave South Birmingham, AL 35294. (jtector@uab.edu).

Supplemental digital content (SDC) is available for this article. Direct URL citations appear in the printed text, and links to the digital files are provided in the HTML text of this article on the journal's Web site (www. transplantjournal.com).

Copyright (C) 2017 Wolters Kluwer Health, Inc. All rights reserved.

ISSN: 0041-1337/17/10104-e86

DOl: $10.1097 / T P .0000000000001646$ preformed antibodies that bind wild type (WT) pig tissues, impeding clinical application of xenotransplantation. ${ }^{1,2}$ Recent advances in nuclease based genome editing have paved the way for the creation of pigs with gene disruptions causing multiple xenoantigen knockouts $(\mathrm{KO})$, whose cells have greatly reduced human antibody binding. ${ }^{3}$ We used CRISPR/Cas9 to create triple gene KO (TKO) pigs whose cells have less antibody binding when exposed to human serum $(\alpha 1$, 3-galactosyltransferase [GGTA1]/cytidine monophosphate-N-acetylneuraminic acid hydroxylase [CMAH]/ b1,4 N-acetylgalactosaminyl transferase [B4GalNT2] KO). ${ }^{4}$ The 3 disrupted genes synthesize carbohydrate xenoantigens..$^{5-7}$ In preclinical pig-to-primate studies, recipients with low levels of xenoreactive antibodies can survive for prolonged periods using $\mathrm{T}$ cell-directed immunosuppression. ${ }^{8}$ Waitlisted patient antibody binding to glycan KO cells compared with class I swine leukocyte antigen (SLA) KO pig cells remains unknown.

This article evaluates the flow cytometric crossmatch (FCXM) of patients on the renal transplant waitlist. Our findings indicate that many patients have a negative threshold FCXM toward the TKO pig. Some of the remaining patients with a significantly positive FCXM have reduced binding to SLA KO class I pig cells. Examining the patterns of human- and pig-reactive IgG suggests that epitopes shared between HLA and SLA molecules may explain this antigenicity. 
A

A

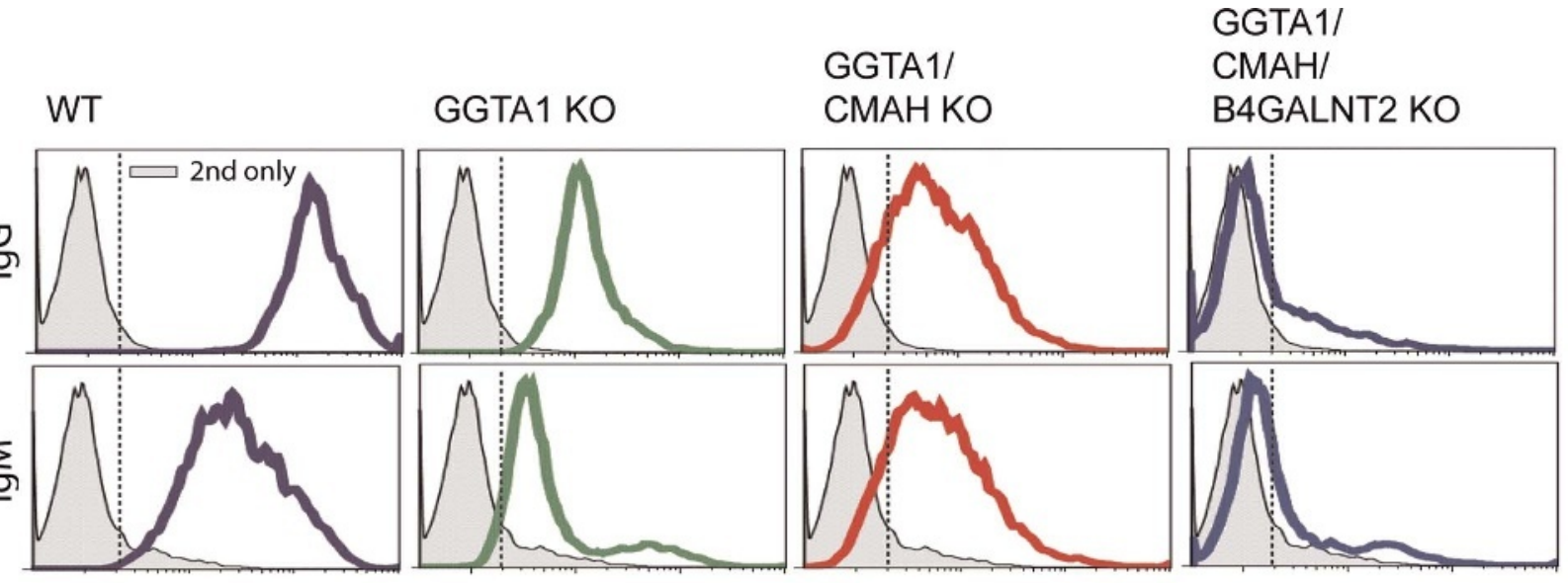

Fluorescence

$\mathrm{B}$
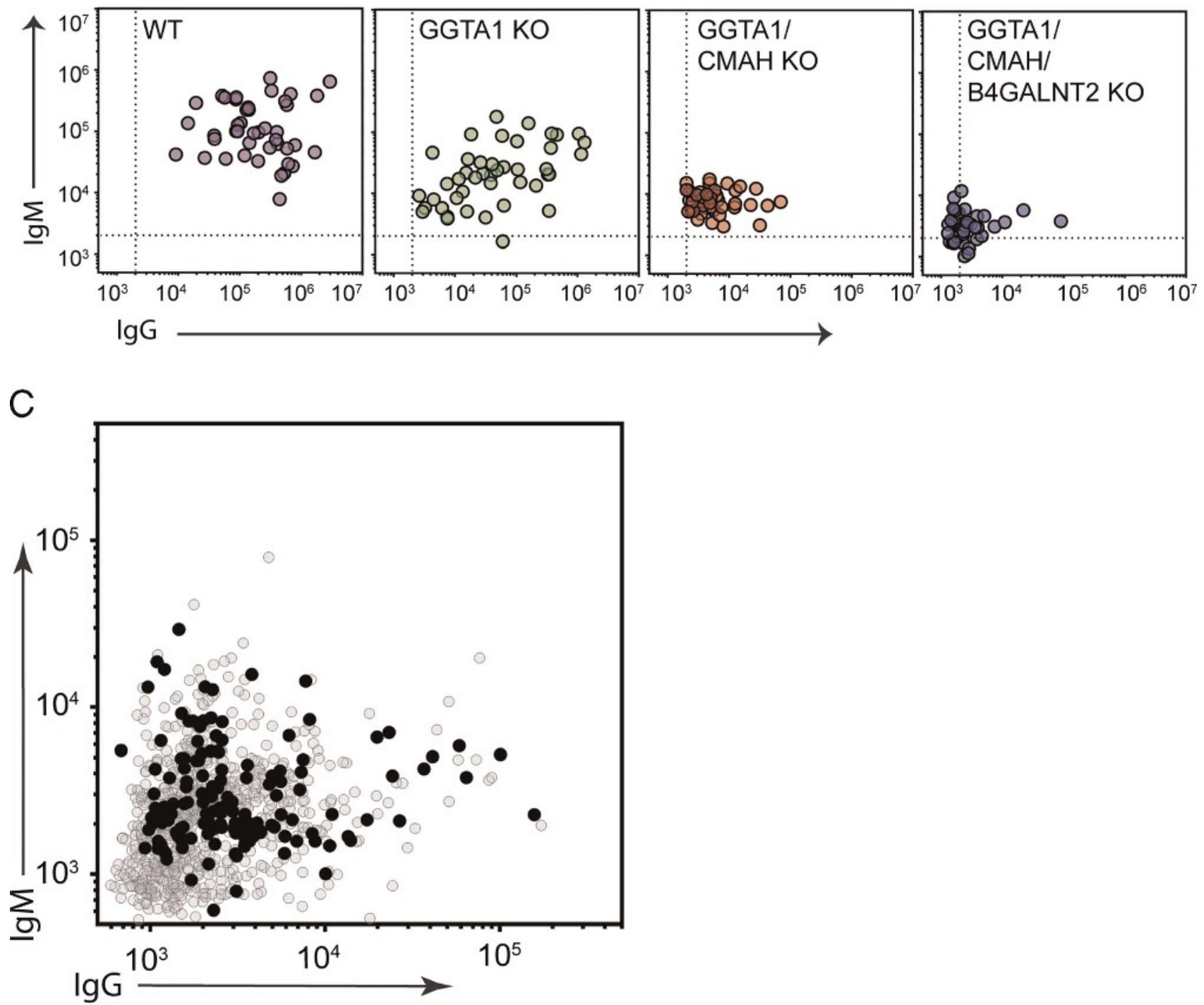

FIGURE 1. Analysis of human immunoglobulin binding to PBMC from genetically modified swine. PBMC were collected, incubated with human serum and analyzed for lgM ( $y$ axes) and lgG ( $x$ axes) binding by flow cytometry. Dotted lines represent an MFI of 2000. A, A single representative patient is screened on WT, GGTA1 KO, GGTA1/CMAH KO, GGTA1/CMAH/B4GalNT2 KO swine PBMC. B, Sera from 44 randomly selected patients with unknown sensitization were incubated with PBMC from all 4 glycan backgrounds. C, Sera from 820 waitlisted renal patient samples were incubated with GGTA1/CMAH/B4GalNT2 knockout PBMC. Solid dots represent 119 samples that were further analyzed in Figure 3. 


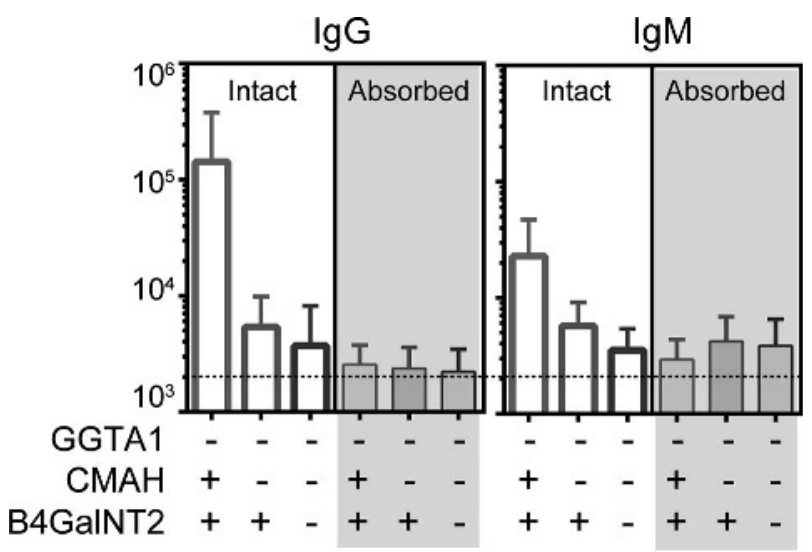

FIGURE 2. Absorption of sera with WT swine RBC minimizes antibody binding to knockout swine PBMC. Porcine-reactive IgG (A) and IgM (B) were depleted from 10 human sera by absorption with WT swine RBC. Immunoglobulin binding to cells deficient in GGTA1, GGTA1/CMAH, or GGTA1/CMAH/B4GalNT2 was analyzed for each serum before (intact) and after (absorbed) absorption. Flow cytometry determined MFIs of antibody binding. Horizontal dotted line appears at MFI 2000. Analysis of depleted sera showed that on average more IgG bound to GGTA1-deficient PBMC than to cells lacking GGTA1/CMAH/B4GalNT2. Wilcoxon matched-pairs rank sum test compared antibody binding preabsorption and postabsorption to each cell type (lgG all comparisons, $P=0.001$; IgM GGTA1 KO, $P=0.001$; GGTA1/CMAH $\mathrm{p}=0.0210$, GGTA1/CM $\mathrm{AH} / \mathrm{B} 4 \mathrm{GalNT2}, P=0.339)$. Dunnett test was performed to compare IgG binding of depleted sera to the different PBMC (GGTA1 vs GGT A1/CMAH, $P=0.923$; GGTA1 vs GGTA1/CMAH/B4GalNT2, $P=0.0239$; GGTA1/CMAH vs GGTA1/CMAH/B4GalNT2, $P=0.307$ ).

\section{MATERIALS AND METHODS}

\section{Animals}

WT and genome edited pigs with GGTA1, GGTA1/CMAH, and GGTA1/CMAH/B4GalNT2 homozygous deletions. ${ }^{4}$ and pigs having GGTA1/SLA-1,-2, and -3 homozygous deletions ${ }^{9}$ were used as peripheral blood mononuclear cells (PBMC) donors. SLA-positive animals all contained identical SLA genotypes (SLA-1*0702, SLA-1*1201, SLA-1*1301, SLA-2*0202, SLA- $2 * 1001$, SLA-3*0402, SLA-3*0502, with the respective accession: AIH07191, AIH07192, AIH07193, AIH07194, AIH07195, AIH07196, AIH07197). RBCs were isolated from WT pigs. Animal work was performed under Indiana University IACUC-approved protocols.

\section{Antibody Binding to Porcine or Human PBMC}

Sera were obtained from deidentified remnant/discarded clinical laboratory samples from patients on the transplant waiting list with Indiana University Institutional Review Board approval. PBMC from unique people were isolated from deidentified/discarded buffy coats, obtained from the Indiana Blood Bank with IRB approval, and used for all binding and elution assays. Bulk PBMCs were analyzed because all porcine PBMC subtypes express the glycans of interest and class I SLA. Swine PBMCs were isolated using Ficoll-Paque Plus (GE-Healthcare, Pittsburgh, PA) and suspended in EX-CELL 610-HSF Serum-Free Medium (Sigma, St. Louis, MO). A 25\% mixture of heatinactivated serum and $2 \times 10^{5}$ cells in EX-CELL with $0.1 \%$ sodium azide was incubated for 30 minutes at $4^{\circ} \mathrm{C}$. Cells were washed 3 times with EX-CELL plus azide and stained with goat antihuman IgG Alexa Fluor 488 and donkey antihuman IgM Alexa Fluor 488 (Jackson
ImmunoResearch Laboratories Inc., West Grove, PA) for 30 minutes at $4^{\circ} \mathrm{C}$. Cells were washed using EX-CELL with azide as above, and flow cytometric analysis completed on BD Accuri C6 flow cytometer.

Use of deidentified remnant/discarded sera, some without sensitization classification, and limited porcine and human cells determined the number of samples and depth of analysis, which could be completed in each assay.

\section{Red Blood Cell Absorption}

Blood was collected, and buffy coat was removed as above from packed red blood cells (RBCs) and washed in phosphate buffered saline. To remove xenoreactive carbohydratespecific human antibodies, an equal volume of packed RBCs was incubated for 30 minutes with human serum at $4^{\circ} \mathrm{C}$. Depleted serum was recovered by high speed centrifugation (5000 RPM) of the RBC/serum mixture. A subset of samples was absorbed with autologous RBCs to assess experimental variability following this process. Seventeen of 18 samples varied by less than $25 \%$, with a single sample changing by $30 \%$ (median fluorescent intensity [MFI], 1181 to 1538 ; calculated as $\%$ variability $=100 \times[$ larger MFI-smaller MFI $] \div$ smaller MFI). Therefore, $25 \%$ variation was chosen as the limit of binding differences that we could detect in the absorption assay. Samples having less than $25 \%$ variability in antibody binding on TKO PBMC cells pre-RBC and post-RBC absorption were selected for further analysis. This subset of samples is used because remaining reactivity must be due to an antigen not on RBCs, but present on nucleated PBMCs.

\section{Porcine-Specific Antibody Elution}

Porcine PBMC and human serum were incubated together for 30 minutes at $4^{\circ} \mathrm{C}$ at $1 \times 10^{6}$ cells per $1-\mu \mathrm{L}$ serum. Cells were washed twice with phosphate buffered saline to remove unbound antibodies. Citric acid/phosphate buffer ( $\mathrm{pH} 3.3$ ) was added for 2 minutes to elute bound antibodies. ${ }^{10}$ After pelleting cells, the supernatant was neutralized with Trisbuffered saline, $\mathrm{pH}$. 8.0, followed by concentration to the predepletion serum volume using Vivaspin $630000 \mathrm{MWCO}$ centrifugal concentrators (Sartorius, New York, NY).

\section{HLA Single-Antigen Screening and SLA Peptide Analysis}

Single-antigen HLA reactivity was measured using LABScreen single-antigen HLA class I beads (One Lambda Inc., Canoga Park, CA). All samples were treated with Dithiothreitol before single-antigen testing for antihuman IgG binding. Bead binding was analyzed on a Luminex Instrument (Luminex, Austin, TX).

\section{RESULTS}

\section{Impact of Glycan Xenoantigen Deletion on FCXM}

Representative single human FCXM show impact of glycan modifications (Figure $1 \mathrm{~A}$ ). IgG and IgM binding to pig PBMC decreased significantly with successive deletion of 3 carbohydrate-modifying genes so that most human sera exhibited between 10-fold and 100-fold reduction in MFI when comparing $\operatorname{IgM}$ and $\operatorname{IgG}$ binding to wild-type and GGTA1/CMAH/B4GalNT2 deficient pigs (Figure 1B). As shown in Figure 1C, sera from 820 transplant-waitlisted patients were screened and $31 \%$ had both IgG and IgM MFIs that were less than 2000. When considering IgG only, $59 \%$ of patients had 
A

IgG
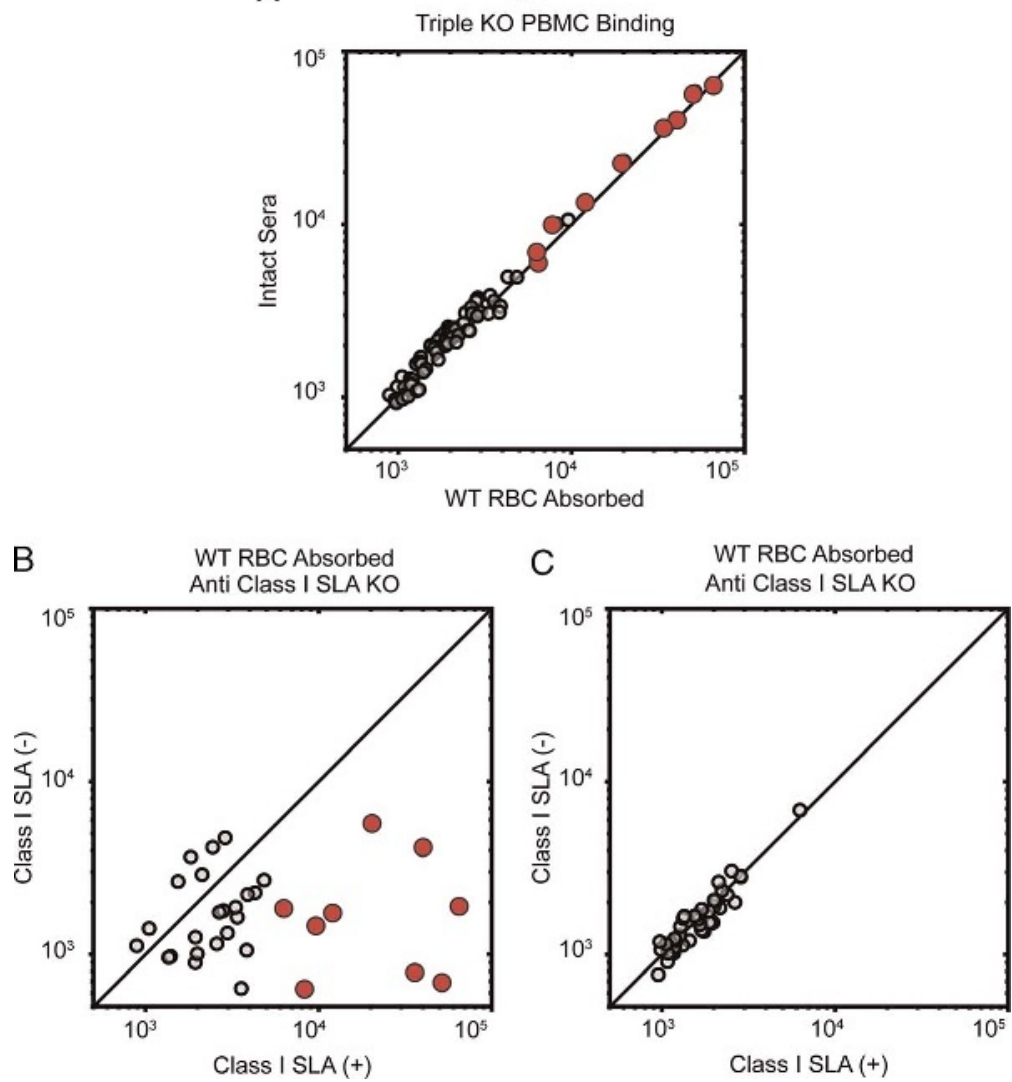

FIGURE 3. Some sera contain antibodies which bind class I SLAs. Flow cytometry evaluated lgG binding from 119 sera to PBMC collected from swine lacking GGTA1/CMAH/B4GalNT2 genes before (intact $y$ axis) and after (absorbed $x$ axis) depletion of antibodies recognizing pig-specific carbohydrate xenoantigens. The preabsorption and postabsorption MFI of 73 sera, shown in panel A, exhibited less than $25 \%$ variation. Forty-six sera demonstrated more that $25 \%$ variation in MFI values and are not shown to simplify analysis. To determine if residual antibodies to TKO swine PBMC-targeted class I SLA molecules, absorbed sera from panel A were incubated with either GGTA1/CMAH/B4GalNT2 knockout ( $x$ axis, class I SLA ${ }^{+}$) or GGTA1/SLA1 knockout ( $y$ axis, class I SLA ${ }^{-}$) swine PBMC. MFI of IgG binding was determined by flow cytometry. Sera exhibiting greater (B) or less (C) than $25 \%$ variation of binding in the presence or absence of class I SLA are shown. Nine sera with high levels of binding to GGTA1/CMAH/B4GalNT2 knockout caused by class I SLA are highlighted (red dots, panels A and B).

MFI $\leq 2000$. MFI of 2000 was selected as threshold MFI as it overlaps with negative control cells incubated with secondary antibodies in the absence of serum in this protocol (Figure 1A). The rest of this study focused on 119 patients who still had antibody binding to PBMC from the TKO pig and sufficient sample for further analysis (Figure 1C, black dots).

\section{Class I SLA Are Xenoantigens}

Many patients on the transplant waitlist have antibodies that target epitopes on the HLA. Therefore, we hypothesized that pig homologs of HLA, the SLA, would be significant xenoantigens in many sensitized patients. HLA and SLA typically have $75 \%$ to $80 \%$ identity to HLA at the amino acid level and have very similar 3D structure. Our class I SLAdeficient pigs were previously characterized, but changes in humoral reactivity to these cells remained unknown. ${ }^{9}$ Immunoglobulin binding to non-SLA xenoantigens was minimized by absorption to WT swine RBCs which do not express SLA molecules. The absorption protocol successfully depleted glycan-specific antibodies from serum and achieved low binding across GGTA1, GGTA1/CMAH, and GGTA1/ CMAH/B4GalNT2 KO cells (Figure 2). Absorption was essential in this experiment to eliminate glycan antibodies and to allow focused evaluation on SLA antigens found on GGTA1/CMAH/B4GalNT2 cells but not on SLA class I/GGTA1 KO cells and allow comparison across backgrounds.

IgG binding to the TKO pig cells before and after absorbing each serum on WT swine RBC was analyzed using flow cytometry. The MFI of 73 sera preabsorption and postabsorption varied less than $25 \%$ (Figure 3A). Ten serum retained dramatically increased levels of binding to TKO cells $\left(\mathrm{MFI}>5 \times 10^{3}\right)$ demonstrating an abundance of reactivity to antigens not on RBCs. To examine this subset of absorbed sera for the presence of class I SLAreactive antibodies, they were incubated with class I SLA expressing TKO PBMC and with class I deficient GGTA1 knockout PBMC. Binding to the different targets varied more than $25 \%$ in some samples (Figure 3B), whereas others demonstrated minimal changes in reactivity despite the presence and absence of class I SLA molecules (Figure 3C). Nine of the 10 high binders showed reduced interaction with SLA KO cells (red dots, Figures 3A and B).

Serum from highly sensitized patients interacts with a broad spectrum of HLA alleles making these patients difficult to transplant. A subset of the 119 samples with known calculated panel reactive antibodies (cPRA) greater than 80 and sufficient sample volume were depleted with WT pig 
A

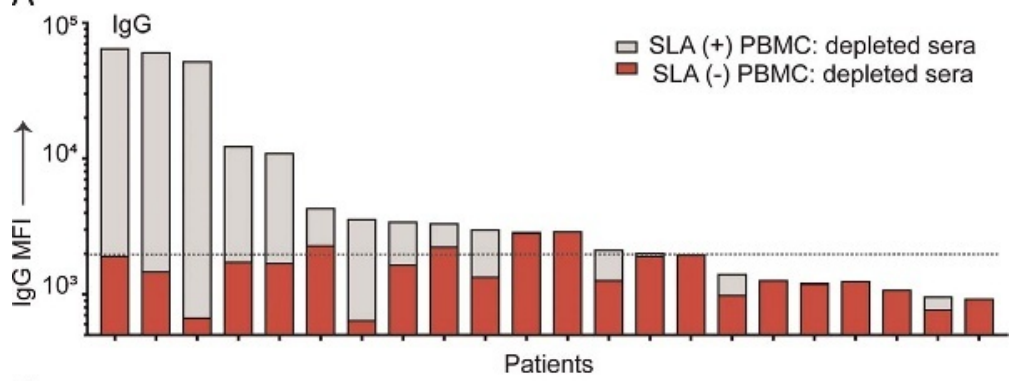

B

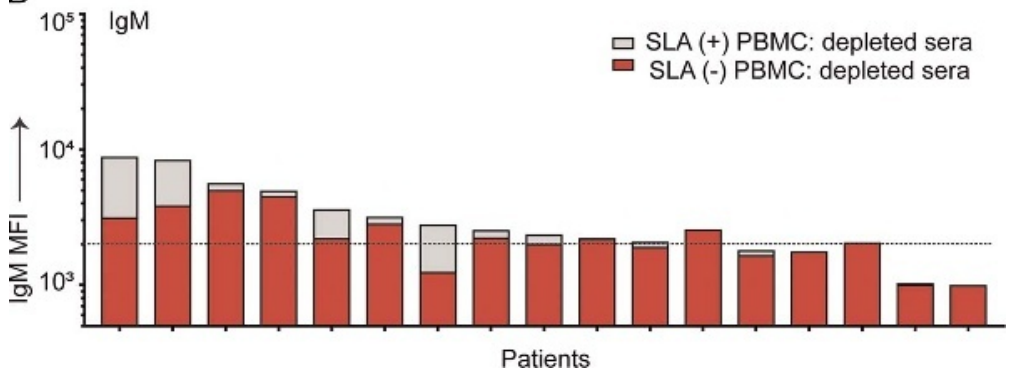

FIGURE 4. Determining the frequency of highly allosensitized human sera containing antibodies Specific for class I SLA molecules. Human sera with panel reactive antibodies greater than 80 were absorbed with WT pig RBC and incubated with PBMC collected from GGTA1 knockout or GGTA1/SLA1 knockout swine. Flow cytometry determined MFls of binding to these cells for lgG (A) and lgM (B). Thirteen of 22 samples contained class I SLA-specific IgG, and 4 of 17 samples, contained lgM that reacted with class I SLA proteins. Horizontal dotted line appears at MFI = 2000 .

RBC and tested on GGTA1/SLA class I-negative and TKO class I-positive cells. Thirteen of 22 samples contained class I SLAspecific $\operatorname{IgG}$ (Figure 4A), and 4 of 17 samples contained lower levels IgM that bound class I SLA proteins (Figure 4B). This demonstrates the presence but not requirement of high levels of SLA reactive antibody in the highly sensitized.

\section{Examining Cross-Reactivity of HLA-Specific IgG With Swine PBMC}

Having shown that deletion of class I SLA reduced binding of human antibodies to swine PBMC, we next sought to determine if human-specific antibodies could bind pig and vice versa. In addition, we compared the HLA allele specificity of antibodies from an individual serum that were eluted from either human or pig cells. A schema describing this process is shown in Figure 5A. We could find sera that had IgG capable of interacting with human and pig PBMC (a representative example is shown in Figure 5B, and a second example is shown in Supplemental Data, SDC, http://links.lww.com/ TP/B391). Reprobing pig PBMC with antibody eluates from human cells showed cross-species reactivity (Figure 5D). Repeating this analysis with $\operatorname{IgG}$ recovered from pig cells on human PBMC verified this result (Figure 5F). Examining the allelic specificity of antibodies eluted from either pig or human PBMC showed that they interacted with overlapping sets of HLA proteins (compare Figures 5E and F). These results demonstrate that antibodies having cross-species reactivity also recognize the same class I HLA antigens.

\section{DISCUSSION}

Antibodies have been a significant barrier to kidney transplantation whether allotransplantation or xenotransplantation. ${ }^{1,11}$ Histocompatibility testing identifies situations in allotransplantation where the recipient has antidonor antibodies to avoid performing transplants with a high risk of antibody-mediated rejection (AMR). ${ }^{11,12}$ In xenotransplantation, once an antigen has been identified, a genome editing strategy can eliminate that antigen and create a more favorable crossmatch. ${ }^{4,13,14}$ This is very encouraging in light of recent data in preclinical pig-to-primate renal xenotransplant studies that show recipients with low levels of xenoreactive antibodies can survive for more than 6 months using $\mathrm{T}$ cell-directed immunosuppression. ${ }^{8,15}$ Avoiding AMR for the patient with a completely negative FCXM (MFI $\leq 2000)$ may be more favorable since we were unable to find completely negative FCXM for the primates.

More than 18000 patients on the United Network of Organ Sharing kidney transplant waitlist are highly sensitized with a cPRA $>80$; these patients have a very difficult time finding a kidney donor with an acceptable crossmatch. Anti-HLA antibodies develop in patients through: blood transfusions, a previous failed renal allograft, or pregnancy. ${ }^{16}$ Donor-specific anti-class I HLA antibodies are associated with inferior renal allograft survival secondary to early AMR. ${ }^{17}$ Patients with cPRA greater than 80 are ideal candidates for initial renal xenotransplant trials, but our data show that the many of these people have anti-class I HLA antibodies that bind our TKO pigs, posing a barrier for their entry into clinical trials (Figure 4). Highly sensitized patients will require further genome editing in the donor pigs before they can be considered for clinical renal xenotransplant trials. The development of reagents to evaluate SLA class II binding is underway, and preliminary evidence suggests that there are some individuals with anti-HLA class II antibodies that cross-react with the SLA II on our TKO pigs (data not shown).

We demonstrated that human- and pig-specific antibodies recognize overlapping sets of HLA alleles, cross-react across species, and bind in bead restricted patterns on HLA beads. 
A
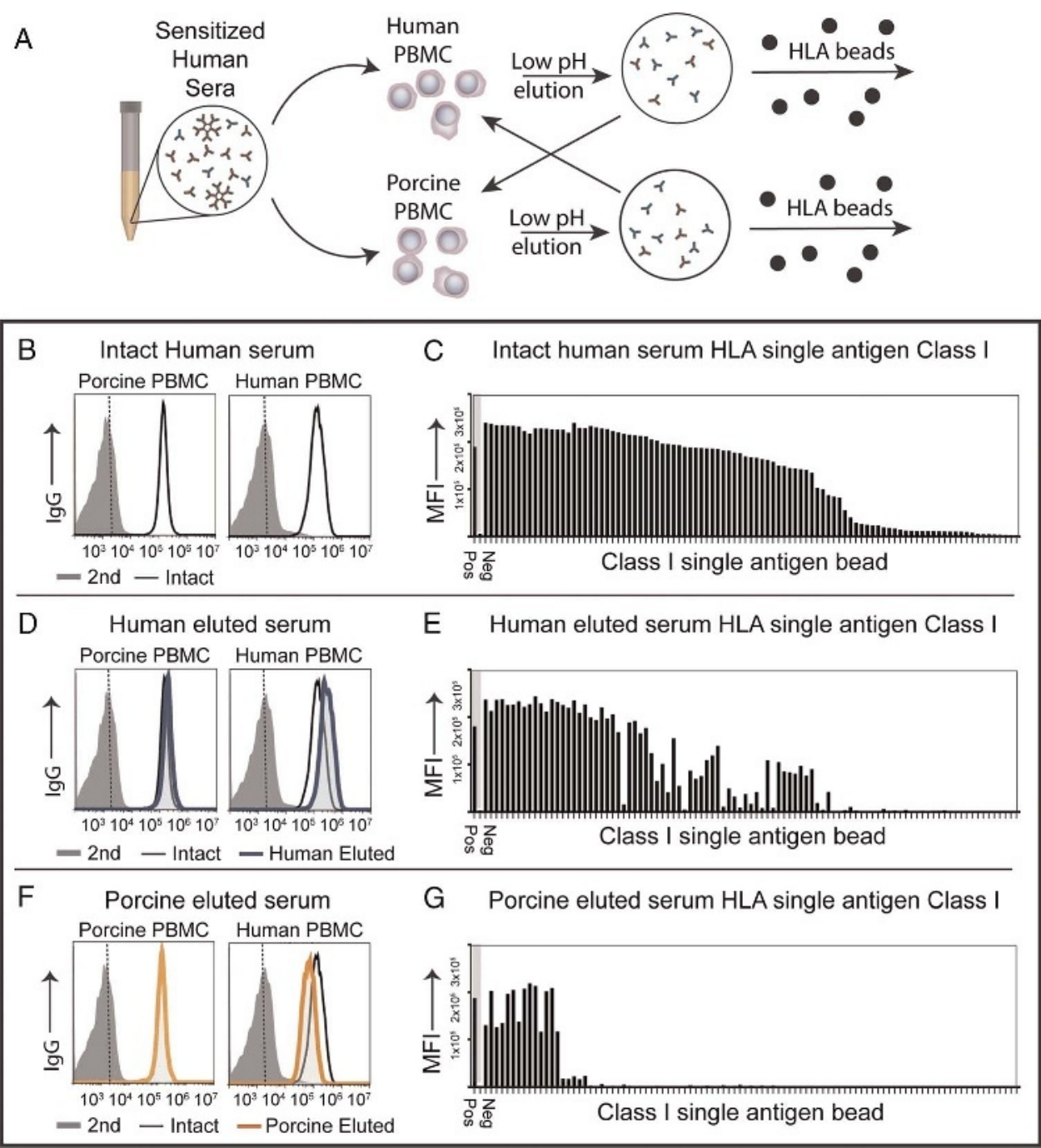

FIGURE 5. Pig-specific human lgG cross-reacts with class I human leukocyte antigens. A, Schema depicting the process of analyzing immunoglobulin cross-reactivity. Flow cytometry determined the MFls of human IgG binding to beads containing a panel of 98 class I HLA proteins. A single representative patient sera is displayed in panels B to G. B, Shows FCXM of intact serum against porcine PBMC and PBMC from a single human. C, IgG from the intact serum broadly reacts with class I HLA proteins as shown by Incubation of the intact serum with 97 class I HLA alleles, each one linked to a specific bead. IgG binding to each bead was probed by flow cytometry and reported as MFI. D, Antibodies were stripped from the human target cells in panel B. Eluted antibodies were tested for reactivity to porcine PBMC and to human cells. Thick blue histograms represent the MFI of eluted antibodies on each target. Solid gray histograms are the secondary only negative controls. E, The human eluted IgG reactivity to HLA beads was tested. F, IgG eluted from Porcine PBMC were tested for reactivity with pig and human cells. Thick orange histograms represent binding of pig eluted antibodies. Solid gray histograms are the secondary only negative controls. G, Antibodies eluted from swine cells recognize a subset of HLA alleles that were recognized by the human-eluted antibodies (compare binding patterns in panels $E$ and $G$ ). HLA single-antigen beads are displayed in the same order from left to right in panels $C, E$, and $G$.

Antibody cross-reactivity of HLA molecules occur at shared common epitopes known as cross-reactive groups (CREGs). ${ }^{18}$ These CREGs drive most humoral-based alloreactivity. The most likely source of class I HLA-related epitopes on pig cells are SLA proteins similarities given their high sequence and structural similarities. A sequence comparison shows that multiple known HLA CREG amino acids can also be found in SLA proteins.

Using a bioinformatics approach, it may be feasible to evaluate all known SLA molecules to find haplotypes with the fewest HLA CREGs so that we can create TKO pigs on that background. That strategy will minimize the need for genome editing of the SLA region to develop pigs with negative crossmatches for the highly sensitized patient. Alternatively, we can genome edit the SLA using clustered interspaced short palindromic repeats/ Cas9 endonuclease on our current genetic background to eliminate the CREGs to evaluate the impact of their deletion on anti-HLA antibody binding. In the unlikely event that neither of these 2 approaches improves the crossmatch of our pigs for highly sensitized patients, we can create SLA I KO pigs with a patient's own HLA allele inserted into the deleted SLA I locus to create a single class I HLA match. These cells have been created and eliminate anti-class I antibody binding in crossmatches, but we have not yet created pigs with these cells (data not shown).

Previous studies have examined the cross-reactivity between HLA-specific antibodies and pig cells, but arrived at conflicting conclusions. HLA-specific monoclonal antibodies that cross-react with SLA molecules have been isolated from humans. ${ }^{19}$ A challenge of the monoclonal antibody approach 
is that limitations of the technology enable analysis of only a few specificities of the potentially enormous antibody pool, making it impossible to determine how abundant SLAreactive antibodies may be in serum. Several reports have provided indirect evidence supporting the idea that HLA-specific immunoglobulin can cross-react with SLA molecules by studying the entire complement of antibodies in serum with elevated panel reactive antibodies after absorbing out "background" xenoantigens. ${ }^{20-23}$ Three studies also relied on the depletion of immunoglobulin using class I-positive SLA platelets to further implicate class I SLA as immunologic targets of these sera. ${ }^{21-23}$ Other studies indicated that sensitization does not increase the levels of porcine-reactive antibodies in human serum. ${ }^{24-26}$ These reports performed their analyses on GGTA1 deficient cells, but did not use absorption to eliminate background xenoreactivity potentially obscuring their ability to detect SLA-specific immunoglobulin.

It has been proposed that the contradictions regarding whether or not class I SLA are xenoantigens may arise from artifacts introduced by the incomplete depletion of background xenoreactive antibodies. ${ }^{25}$ The assays performed in Figure 5 avoided this issue by examining the pattern of HLA binding in antibodies that bound to and were eluted from both PBMC taken from both humans and SLApositive swine PBMC. Human and pig cells eluted antibodies bind disparate HLA subsets and signify that the cell-antibody interactions were driven by targeted binding of epitopes and not nonspecific adherence of immunoglobulin through Fc receptors or other means. The WT pig RBC absorption protocol used markedly reduced non-SLA antigenicity (Figure 2). Further, when analyzing absorbed sera, we showed that GGTA1-deficient SLA-KO cells exhibited less antibody binding than SLA-positive TKO PBMC (Figure 3). Incomplete or absent absorption would eliminate this result and obscure SLA binding.

The prospects for using xenotransplantation to eliminate the shortage of donor kidneys have improved dramatically. In addition, the path forward to eliminate class I SLA xenoantigens is clear, and the technology to create the necessary pigs is readily available. We are now entering a time where we can plan to initiate clinical trials for patients with a negative crossmatch, and it is conceivable that donor pigs can be created for every patient on a transplant waitlist that should be free from the threat of early AMR.

This work begins to establish a roadmap for genome editing strategies to create pigs that can be used safely in highly sensitized patients, opening up the possibility of transplanting every patient on the waitlist.

\section{REFERENCES}

1. Cooper DK. Xenoantigens and xenoantibodies. Xenotransplantation. 1998;5:6-17.

2. Byrne GW, McGregor CG, Breimer ME. Recent investigations into pig antigen and anti-pig antibody expression. Int J Surg. 2015;23(Pt B): 223-228.

3. Wang ZY, Li P, Butler JR, et al. Immunogenicity of renal microvascular endothelial cells from genetically modified pigs. Transplantation. 2016;100: 533-537.
4. Estrada JL, Martens G, Li P, et al. Evaluation of human and non-human primate antibody binding to pig cells lacking GGTA1/CMAH/ß4GalNT2 genes. Xenotransplantation. 2015;22:194-202.

5. Byrne GW, Stalboerger PG, Du Z, et al. Identification of new carbohydrate and membrane protein antigens in cardiac xenotransplantation. Transplantation. 2011;91:287-292.

6. Oriol R, Ye Y, Koren E, et al. Carbohydrate antigens of pig tissues reacting with human natural antibodies as potential targets for hyperacute vascular rejection in pig-to-man organ xenotransplantation. Transplantation. 1993; 56:1433-1442.

7. Salama A, Evanno G, Harb J, et al. Potential deleterious role of antiNeu5Gc antibodies in xenotransplantation. Xenotransplantation. 2015; 22:85-94.

8. Higginbotham L, Mathews D, Breeden CA, et al. Pre-transplant antibody screening and anti-CD154 costimulation blockade promote long-term xenograft survival in a pig-to-primate kidney transplant model. Xenotransplantation. 2015;22:221-230.

9. Reyes LM, Estrada JL, Wang ZY, et al. Creating class I MHC-null pigs using guide RNA and the Cas9 endonuclease. J Immunol. 2014;193: 5751-7.

10. Storkus WJ, Zeh HJ 3rd, Salter RD, et al. Identification of T-cell epitopes: rapid isolation of class I-presented peptides from viable cells by mild acid elution. J Immunother Emphasis Tumor Immunol. 1993;14:94-103.

11. Tambur A, Claas F. HLA epitopes as viewed by antibodies: what is it all about? Am J Transplant. 2015:15:1148-1154.

12. Terasaki Pl. Humoral theory of transplantation. Am J Transplant. 2003;3: 665-673.

13. Burlak C, Paris LL, Lutz AJ, et al. Reduced binding of human antibodies to cells from GGTA1/CMAH KO pigs. Am J Transplant. 2014;14: 1895-900.

14. Li P, Estrada JL, Burlak C, et al. Efficient generation of genetically distinct pigs in a single pregnancy using multiplexed single-guide RNA and carbohydrate selection. Xenotransplantation. 2015;22:20-31.

15. Iwase H, Liu H, Wijkstrom M, et al. Pig kidney graft survival in a baboon for 136 days: longest life-supporting organ graft survival to date. Xenotransplantation. 2015;22:302-309.

16. Patel R, Terasaki PI. Significance of the positive crossmatch test in kidney transplantation. N Engl J Med. 1969;280:735-739.

17. Lefaucheur C, Loupy A, Hill GS, et al. Preexisting donor-specific HLA antibodies predict outcome in kidney transplantation. J Am Soc Nephrol. 2010;21:1398-406.

18. Duquesnoy RJ, Marrari M, Mulder A, et al. First report on the antibody verification of HLA-ABC epitopes recorded in the website-based HLA Epitope Registry. Tissue Antigens. 2014;83:391-400.

19. Mulder A, Kardol MJ, Arn JS, et al. Human monoclonal HLA antibodies reveal interspecies crossreactive swine $\mathrm{MHC}$ class I epitopes relevant for xenotransplantation. Mol Immunol. 2010;47:809-815.

20. Oostingh GJ, Davies HF, Tang KC, et al. Sensitisation to swine leukocyte antigens in patients with broadly reactive HLA specific antibodies. Am J Transplant. 2002;2:267-273.

21. Varela ID. Cross-reactivity between swine leukocyte antigen and human anti-HLA-specific antibodies in sensitized patients awaiting renal transplantation. J Am Soc Nephrol. 2003;14:2677-2683.

22. Taylor CJ, Tang KG, Smith SI, et al. HLA-specific antibodies in highly sensitized patients can cause a positive crossmatch against pig Lymphocytes. Transplantation. 1998;65:1634-41.

23. Naziruddin B, Durriya S, Phelan D, et al. HLA antibodies present in the sera of sensitized patients awaiting renal transplant are also reactive to swine leukocyte antigens. Transplantation. 1998;66: 1074-80.

24. Bartholomew A, Latinne D, Sachs DH, et al. Utility of xenografts: lack of correlation between PRA and natural antibodies to swine. Xenotransplantation. 1997;4:34-9.

25. Wong BS, Yamada K, Okumi M, et al. Allosensitization does not increase the risk of xenoreactivity to alpha1,3-galactosyltransferase gene-knockout miniature swine in patients on transplantation waiting lists. Transplantation. 2006;82:314-9.

26. Hara H, Ezzelarab M, Rood PP, et al. Allosensitized humans are at no greater risk of humoral rejection of GT-KO pig organs than other humans. Xenotransplantation. 2006;13:357-65. 